\title{
Dynamic switch of immunity and antitumor effects of metformin in rat spontaneous esophageal carcinogenesis
}

\author{
Ryohei Takei ${ }^{1,3}$ (]) $\cdot$ Tomoharu Miyashita $^{1,2} \cdot$ Satoshi Takada ${ }^{1} \cdot$ Hidehiro Tajima $^{1} \cdot$ Itasu Ninomiya $^{1} \cdot$ \\ Hiroyuki Takamura ${ }^{1,2}$. Sachio Fushida ${ }^{1} \cdot$ Ai Harashima $^{3}$ - Seiichi Munesue ${ }^{3} \cdot$ Shintaro Yagi $^{1} \cdot$ Noriyuki Inaki $^{1}$. \\ Tetsuo Ohta ${ }^{1}$. Yasuhiko Yamamoto ${ }^{3}$
}

Received: 20 February 2021 / Accepted: 29 July 2021 / Published online: 16 August 2021

(c) The Author(s) 2021

\begin{abstract}
Chronic inflammation contributes to tumor development by creating a local microenvironment that facilitates neoplastic transformation and potentiates the progression of cancer. Esophageal cancer (EC) is an inflammation-associated malignancy with a poor prognosis. The nature of the switch between chronic inflammation of the esophagus and EC-related immunological changes remains unclear. Here, we examined the dynamic alterations of immune cells at different stages of chronic esophagitis, Barrett's esophagus (BE) and EC using an esophageal spontaneous carcinogenesis rat model. We also investigated the anticancer effects of metformin. To stimulate EC carcinogenesis, chronic gastroduodenal reflux esophagitis via esophagojejunostomy was induced in 120 rats in metformin-treated and non-treated (control) groups. After 40 weeks, BE and EC developed in $96.7 \%$ and $63.3 \%$ of the control group, and in $66.7 \%$ and $23.3 \%$ of the metformin-treated group, respectively. Flow cytometric analysis demonstrated that the balance of M1/M2-polarized or phospho-Stat3-positive macrophages, regulatory T, cytotoxic T, natural killer (NK), NK T cells, and Th17 T cells was dynamically changed at each stage of the disease and were resolved by metformin treatment. These findings clarify the immunity in esophageal carcinogenesis and suggest that metformin could suppress this disease by improving the immunosuppressive tumor microenvironment and immune evasion.
\end{abstract}

Keywords Gastroduodenal reflux esophagitis - Esophageal cancer · Chronic inflammation · Tumor microenvironment . Metformin

\section{Introduction}

Esophageal cancer (EC) is an aggressive cancer with a poor prognosis. More than $90 \%$ of EC cases are histologically comprised of esophageal squamous cell carcinoma (ESCC). The incidence of esophageal adenocarcinoma (EAC) has

Ryohei Takei

ryohei_20@stu.kanazawa-u.ac.jp

Yasuhiko Yamamoto

yasuyama@med.kanazawa-u.ac.jp

1 Department of Gastroenterologic Surgery, Kanazawa University Graduate School of Medical Sciences, Kanazawa 920-8640, Japan

2 Department of Surgical Oncology, Kanazawa Medical University Hospital, 13-1 Takaramachi, Kanazawa 920-8640, Japan

3 Department of Biochemistry and Molecular Vascular Biology, Kanazawa University Graduate School of Medical Sciences, Kanazawa 920-8640, Japan recently increased dramatically in the USA and several Western countries [1].

EAC generally originates from Barrett's esophagus (BE) and is pathologically characterized by intestinal metaplasia of the distal esophagus, followed by gastroesophageal reflux disease (GERD) and subsequent chronic esophagitis. Chronic esophagitis was reported to result in a 4.5- and 29.8-fold increase in the relative risk of developing EAC and BE, respectively [2].

Carcinogenesis is caused by chronic inflammation and the production of reactive oxygen species, leading to oncogenic mutations [3]. Previous studies have reported somatic mutations in cancer-related genes in EAC, such as TP53 [4], TP16 [5], SMAD4 [6], PIK3CA [7], EGFR [8], and APC [9]. Additionally, chronic inflammation forms a cancer microenvironment that can induce the proliferation of cancer cells and enhance their survival. Therefore, inflammatory responses in cancer are a two-edged sword, with tumor-suppression (cancer-killing) and tumor-promoting dynamic changes in immune cell populations. The tumor-suppressive and 
cancer-killing cell types include natural killer (NK) cells, NK T (NKT) cells, CD8 ${ }^{+} \mathrm{T}$ lymphocytes, and M1-polarized macrophages (M1Ms). In contrast, regulatory T cells (Tregs) and M2-polarized macrophages (M2Ms) are anti-inflammatory cell types that promote tumor formation in the cancer microenvironment. Inflammation can also activate intracellular signaling proteins, such as nuclear factor-kappa B (NF-KB) and signal transducer and activator of transcription 3 (STAT3) via inflammation mediators that include interleukin-6 (II-6). Activated STAT3 plays an important role in tumor growth and survival [10], and in the suppression of antitumor immunity by enhancing the activity of Tregs and M2Ms [11, 12].

Our Gastroenterologic Surgery group previously established an esophageal spontaneous carcinogenesis rat model, which shows symptoms similar to those of human EC, by inducing gastroduodenal reflux esophagitis after performing an esophagojejunostomy [13]. This rat model allowed us to examine changes in the composition of esophageal immune cells at all stages of esophageal carcinogenesis, including proliferative hyperplasia (PHP), BE, ESCC, and EAC, to clarify the mechanisms underlying carcinogenesis stimulation in EAC through the inflammatory reaction.

Metformin is a drug used to treat type 2 diabetes. The drug also has anti-hyperglycemic and unexpected anticancer effects. Epidemiological studies have associated metformin with a reduction in the incidence and mortality rates of many solid tumors [14]. Clinical evaluation of the chemopreventive and anti-neoplastic effects of metformin is ongoing in phase III trials for several cancers, including endometrial cancer, colorectal cancer, and breast cancer [15]. The anticancer mechanisms of metformin include the activation of AMP kinase (AMPK), which can preferentially kill cancerinitiating/stem cells of glioblastoma, breast, and ovarian cancers [16-18]. Furthermore, the inactivation of STAT3 by metformin reportedly inhibits cell growth and induces apoptosis in esophageal, pancreatic, and breast cancers [19-21].

The aims of this study were to elucidate the dynamic changes in immune cell populations in the esophagus during the carcinogenic transition from GERD to Barrett's metaplasia and finally to EC. Additionally, the preventive effects of metformin on esophageal carcinogenesis were investigated by evaluating the esophageal microenvironment using our rat surgical model of duodenogastroesophageal reflux (DGER).

\section{Materials and methods}

\section{Experimental animals and metformin treatment}

Sprague-Dawley male rats (4 weeks old) weighing $\sim 250 \mathrm{~g}$ were used for all experiments. The animals were housed at a constant temperature of $22 \pm 3{ }^{\circ} \mathrm{C}$ and $55 \pm 5 \%$ humidity under a 12-h light-dark cycle. The rats were fed standard solid chow (CRF-1; Charles River Laboratories, Yokohama, Japan) and tap water. Following a 24-h fast, an upper abdominal incision and esophagojejunostomy were made under isoflurane inhalation anesthesia to induce DGER. The animals were divided into three groups. The sham-operated control group $(n=5)$ underwent only laparotomy and the rats were fed standard chow. The control group $(n=60)$ underwent esophagojejunostomy and were fed standard chow (CRF-1). The metformin-treated group $(n=60)$ underwent esophagojejunostomy and were fed CRF-1 containing 1.3\% metformin $(900 \mathrm{mg} / \mathrm{kg} / \mathrm{day})$. The dosage of metformin was based on a non-clinical study of the drug toxicity performed by Sumitomo Dainippon Pharma Corporation (Osaka, Japan). Metformin was provided by Sumitomo Dainippon Pharma Corporation and Towa Pharmaceutical Corporation (Osaka, Japan). At $10(n=10), 20(n=10), 30(n=10)$, and $40(n=30)$ weeks after the operation, the rats were subjected to laparotomy and thoracotomy under isoflurane inhalation for the extraction of specimens. After placing a $24 \mathrm{G}$ needle into the left ventricle and making a small incision in the right auricle, whole perfusion was performed with normal saline. After the animals were sacrificed by deep inhalation anesthesia, the entire esophagus and anastomosed jejunum were extracted for histopathological and flow cytometry analyses. This study was approved by the Institutional Animal Care and Use Committee of the Graduate School of Medical Sciences, Kanazawa University (AP-153540; Kanazawa, Japan).

\section{Pathological assessment}

Extracted tissues were fixed in 10\% neutral-buffered formaldehyde for at least $24 \mathrm{~h}$. After fixation, the specimens were cut at 3-mm intervals along the long axis and embedded in paraffin. Each paraffin block was sliced into $5-\mu \mathrm{m}$ sections and stained with hematoxylin and eosin. Histological findings in the specimens were classified into the following three categories according to a previous report [23]:

1. Squamous proliferative hyperplasia (PHP), which features thickening of the epithelium to twice that of a normal epithelium with acanthosis, elongation of the papillae, and parakeratosis. Other features include thickening of the basal layer of the squamous epithelium and preservation of a stratified appearance.

2. Barrett's metaplasia (BM), which features replacement of the esophageal squamous epithelium with columnarlined epithelium comprised of gastric and/or intestinal cells.

3. Carcinoma, featuring cellular, and structural atypism with epithelial invasion into the submucosal layer. EAC shows dysplastic glandular cell growth with both atypia 
and invasiveness. ESCC is a type of squamous cell dysplasia with marked cellular and structural atypism.

For immunohistochemical studies, the sections were deparaffinized, rehydrated, and then treated with citrate buffer (pH 6.7) at $95{ }^{\circ} \mathrm{C}$ for $20 \mathrm{~min}$. Following endogenous peroxidase blocking with $3 \%$ hydrogen peroxide, the sections were incubated overnight at $4{ }^{\circ} \mathrm{C}$ with specific antibody to pStat3 (1:100; anti-Stat3 [phospho S727], ab30647; Abcam, Cambridge, UK). The slides were then incubated with horseradish peroxidase (HRP)-labeled anti-rabbit or anti-mouse IgG (Dako, Foster City, CA, USA) for $1 \mathrm{~h}$. Immunostaining was visualized by incubation with 3,3-diaminobenzidine tetrahydrochloride (DAB; Dako) for 1-2 min and counterstained with Meyer hematoxylin for $30 \mathrm{~s}$. Negative controls were prepared by replacing the primary antibodies with buffer solution.

\section{Isolation of immune cells}

The proximal or distal esophagus of anastomosed rats was dissected and flushed with Dulbecco's modified Eagle's medium (DMEM). The tissue was minced with scissors and placed in a $50 \mathrm{~mL}$ tube with $10 \mathrm{~mL}$ collagenase solution (DMEM supplemented with $1 \mu \mathrm{g} / \mathrm{mL}$ type 2 collagenase and $1 \mu \mathrm{g} / \mathrm{mL}$ DNase I; Sigma-Aldrich, St. Louis, MO, USA). Following incubation for $30 \mathrm{~min}$ at $37^{\circ} \mathrm{C}$ with gentle shaking, $10 \mathrm{~mL}$ DMEM containing 10\% fetal bovine serum (FBS) was added to the collagenase solution. Isolated cells were strained through a $40 \mu \mathrm{m}$ filter and washed twice with fluorescence-activated cell sorting (FACS) buffer (PBS containing $2 \%$ FBS). Cells were then resuspended in $600 \mu \mathrm{L}$ FACS buffer for analysis.

\section{Flow cytometry analyses}

After treatment with Fc block (anti-rat CD16/CD32 antibody; BD Biosciences, San Jose, CA, USA), the cells were incubated with antibodies specific to fluorescein isothiocyanate (FITC)-conjugated anti-rat CD3, phycoerythrin (PE)-Cy7-conjugated anti-rat CD4, PE-conjugated anti-rat CD8a, adenomatous polyposis coli (APC)-conjugated antirat NK1.1 (BioLegend, San Diego, CA, USA), PE-conjugated anti-rat CD25 (eBioscience, Waltham, MA, USA), PE-Cy7-conjugated anti-rat CD11b/c (BD Bioscience), FITC-conjugated anti-rat CD68 (AbD Serotec, Raleigh, NC, USA), PE-conjugated anti-rat CD86 (BioLegend), and APCconjugated anti-rat CD163 (AbD Serotec). Dead cells were excluded from flow cytometry analysis by adding propidium iodine solution. Cell numbers were evaluated using the BD Cell Viability kit (BD Biosciences). For the detection of Tregs, intracellular FoxP3 staining was performed using an anti-rat FoxP3-APC staining kit (eBioscience) according to the manufacturer's instructions. To detect intracellular cytokines, $1 \mathrm{~h}$ brefeldin A (BD Biosciences) treatment and surface marker staining were performed, followed by fixation and permeabilization with the Cytofix/CytoPerm kit (BD Biosciences). Samples were then stained with specific antibodies for eFlour660-conjugated anti-rat tumor necrosis factor-alpha (TNF- $\alpha$; eBioscience), Alexa647-conjugated anti-rat interferon-gamma (IFN- $\gamma$; BioLegend), Alexa647conjugated anti-rat IL-10 (BD Biosciences), Alexa405conjugated anti-rat transforming growth factor-beta (TGF- $\beta$; Novus Biologicals, Centennial, CO, USA), PE-conjugated anti-rat IL-17 (Life Technologies, Carlsbad, CA, USA), and Alexa647-conjugated anti-rat Stat3 (pTyr705 and pSer727; BD Biosciences). Flow cytometry analysis was performed using FACSAria Fusion (BD Bioscience). The obtained data were re-analyzed using FlowJo v 9.6.1 software (FlowJo, Ashland, OR, USA). Unstained, single stains, and fluorescence minus one (FMO) controls were used to set compensation and gates.

\section{Statistical analyses}

Statistical analyses were performed using Student's $t$-test or ANOVA with the Tukey-Kramer post-hoc test using Ekuseru-Toukei 2015 software (Social Survey Research Information Co., Ltd., Japan). $P<0.05$ was considered statistically significant.

\section{Results}

\section{Chronological evaluation of changes in immune cell characteristics during inflammation and carcinogenesis}

In the esophageal spontaneous carcinogenesis model, esophagitis spreads macroscopically from the anastomotic region to the oral side of the esophagus with irregular wall thickness and dilatation over time (Fig. 1). Intestinal metaplasia-like lesions were observed without a clear demarcation between the esophagus and jejunum 20 weeks after esophagojejunostomy (Fig. 1c, upper panel). Overt EC was observed as an ulcer or mass $\sim 40$ weeks post-surgery (wps) (Fig. 1d, upper panel). Histologically, squamous PHP and slight thickening of the lamina propria were observed in all the animals at 10 wps (Fig. 1b, lower panel). These hyperplastic changes became more severe. BM was evident in some rats at 20 wps and EAC and ESCC were observed at $\sim 40$ wps (Fig. 1c, d). The infiltration of immune cells, mostly small and round cells, such as lymphocytes, into the epithelium and lamina propria of the esophagus was observed in the early stage at 10 wps (Fig. 1b). Infiltration 


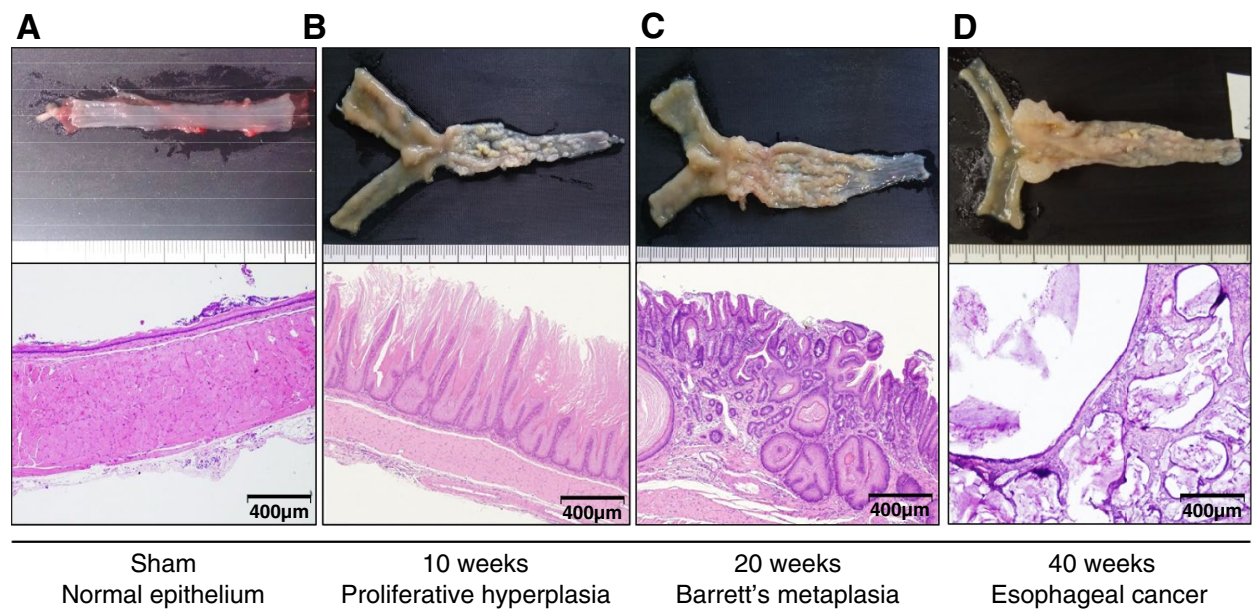

Fig. 1 a Sham-operated control rats (Sham). Normal epithelium. b A distal esophagus at 10 weeks after the operation (10 weeks). The esophagus was macroscopically widened and thickened by chronic gastroduodenal content reflux esophagitis, squamous proliferative hyperplasia (PHP) and slight thickening of the lamina propria were microscopically observed. Magnification, $\times 10$. c A distal esophagus at 20 weeks after the operation (20 weeks). Intestinal metaplasia-like lesions were observed without a sharp demarcation between esopha-

became more severe at 20 wps (Fig. 1c). Quantitative flow cytometry revealed markedly increased $\mathrm{CD}^{+} \mathrm{T}$ lymphocytes and $\mathrm{CD} 11 \mathrm{~b}^{+} \mathrm{CD} 68^{+}$macrophage numbers in esophageal lesions (Fig. 2a, b). Increases of 7.4- and 16.5-fold, respectively, were evident compared to those in the shamoperated control group at 20 weeks (Fig. 2a, b). Thereafter, the infiltration of these cells declined (Fig. 2a, b). A similar pattern of cell infiltration was also observed in NK and NKT cells (Fig. 2c). In terms of the content of $\mathrm{CD}^{+} \mathrm{T}$ lymphocytes, the percentage of $\mathrm{CD}^{+} \mathrm{T}$ cells increased from 27\% (sham-control group) to 67\% (control group) (Fig. 2d). Surprisingly, the percentage of $\mathrm{CD} 8^{+}$cells increased from $0.7 \%$ (sham-control group) to $27 \%$ (control group) during the observation period (Fig. $2 \mathrm{~d}$ ). $\mathrm{CD} 11 \mathrm{~b}^{+} \mathrm{CD} 68^{+} \mathrm{CD} 163^{+}$ M2M macrophages were more predominant in shamoperated esophagus samples (Fig. 2e). However, the $\mathrm{CD} 11 \mathrm{~b}^{+} \mathrm{CD} 68^{+} \mathrm{CD} 163^{-} \mathrm{M} 1 \mathrm{M}$ macrophage ratio increased after surgery, peaked at $20 \mathrm{wps}$, and decreased thereafter (Fig. 2e).

\section{Metformin treatment inhibits development of BE and EC}

We next investigated the effects of metformin using this animal model. Compared to untreated controls, metformin treatment markedly attenuated wall thickening and changes in the dilation of the esophagus (Fig. 3a). The frequencies and sizes of $\mathrm{BE}$ and $\mathrm{EC}$ lesions were substantially attenuated after metformin treatment (Fig. 3a, b). Microscopic observations revealed the beneficial effects on esophagitis and the gus and jejunum. Pathologically, the hyperplastic changes became severer and Barrett's metaplasia were evident in some rats. Magnification, $\times 10$. d A distal esophagus at 40 weeks after the operation (40 weeks). Overt esophageal cancer was seen as an ulcer or a mass at 30 to 40 weeks. Pathologically, esophageal adenocarcinoma (EAC) and esophageal squamous cell carcinoma were observed. Magnification, $\times 10$

development of BE and EC lesions in the metformin-treated group compared to those in the untreated control (Fig. 3a). Quantitative analyses demonstrated that $\mathrm{BE}$ was present in $0, \sim 50, \sim 70$, and $96.7 \%$ of subjects, and EC was observed in $0,0, \sim 30$, and $66.7 \%$ of subjects at $10,20,30$, and $40 \mathrm{wps}$, respectively, in non-treated controls (Fig. 3b). In contrast, the metformin-treated group showed a lower incidence of $\mathrm{BE}(0, \sim 20, \sim 30$, and $66.7 \%)$ and $\mathrm{EC}(0,0, \sim 10$, and $23.3 \%)$ at 10, 20, 30, and 40 wps, respectively (Fig. 3b). However, we did not observe any differences in the incidence of EAC and ESCC between the metformin-treated and untreated control groups at $40 \mathrm{wps}$ (Fig. 3c). Accordingly, we tentatively defined the "inflammatory phase" as $10 \mathrm{wps}$, "Barrett's metaplasia rising phase" as $20 \mathrm{wps}$, and "carcinogenesis phase" as 40 wps.

\section{Dynamic alterations in immune cell characteristics during inflammation and carcinogenesis, and response to metformin}

Flow cytometry analyses were performed to characterize infiltrated immune cell populations in esophageal tissues during the inflammatory phase (10 wps), Barrett rising phase (20 wps), and carcinogenesis phase (40 wps) of the metformin-treated group and the non-treated controls. First, we focused on the T cell population (Fig. 4). The population of infiltrated $\mathrm{CD}^{+} \mathrm{T}$ cells peaked at $20 \mathrm{wps}$, but metformin did not affect its infiltration dynamics (Fig. 4a, b). However, metformin treatment significantly increased $\mathrm{CD}^{+} \mathrm{T}$ cell numbers at 10 and 40 wps (Fig. 4b). In the inflammatory 
Fig. 2 a-c Flow cytometric analyses of the number of $\mathrm{CD}^{+} \mathrm{T}$ lymphocytes, pan macrophages $\left(\mathrm{CD} 3^{-} \mathrm{CD} 11 \mathrm{~b}^{+} \mathrm{CD} 68^{+}\right), \mathrm{NK}$ cells $\left(\mathrm{CD} 3^{-} \mathrm{NK} 1.1^{+}\right)$, and NKT cells $\left(\mathrm{CD}^{+} \mathrm{NK} 1.1^{+}\right)$in sham-operated control rats (Sham) and in rats at 10, 20, and 40 weeks after the surgery $(10 \mathrm{~W}, 20 \mathrm{~W}$ and, $40 \mathrm{~W}$, respectively). $\mathbf{d}$ The proportions of $\mathrm{CD}^{+}$and $\mathrm{CD}^{+} \mathrm{T}$ cells in $\mathrm{CD}^{+} \mathrm{T}$ cells. e The proportions of $\mathrm{CD} 11 \mathrm{~b}^{+} \mathrm{CD} 68^{+} \mathrm{CD} 86^{+}$ M1-like (M1Ms) and

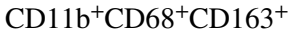
M2-like (M2Ms) macrophages in pan macrophages. Data are presented as the mean $\pm \mathrm{SEM}$; $\mathrm{n}=6$ per group; *, $p<0.05$; **, $p<0.01 ; * * *, p<0.001$
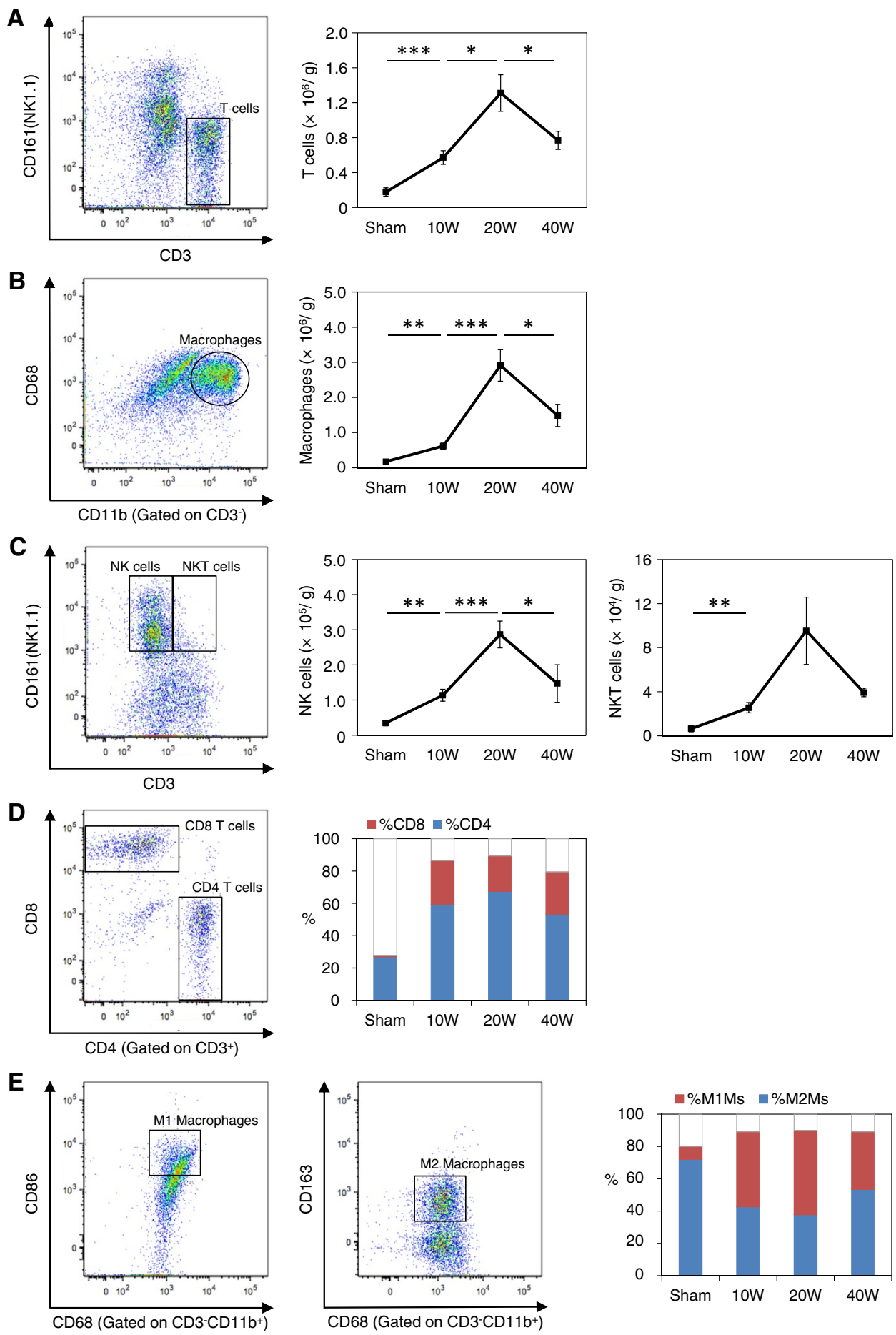

phase at 10 wps, the numbers of $\mathrm{CD} 4^{+}$and $\mathrm{CD} 8^{+} \mathrm{T}$ cells were significantly increased by metformin treatment in the esophagus (Fig. $4 \mathrm{~d}-\mathrm{f}$ ). The increased $\mathrm{CD} 4^{+} \mathrm{T}$ cell populations at 10 weeks were $\mathrm{CD} 4^{+} \mathrm{CD} 25^{+} \mathrm{Foxp} 3^{+}$Treg cells and CD $4^{+}$NK1.1 ${ }^{-}$IL-17A ${ }^{+}$Th17 cells (Fig. 4g, h). We did not observe any significant changes in the expression of TNF- $\alpha$ and INF- $\gamma$ in $\mathrm{CD}^{+}{ }^{+}$and $\mathrm{CD} 8^{+} \mathrm{T}$ cells, in the $\mathrm{CD} 4 / \mathrm{CD} 8$ ratio, or in the percentage of TGF- $\beta^{+} \mathrm{IL}-10^{+}$Tregs at 10 weeks (Fig. 4c, i-k).

During the transition from the inflammatory phase to the carcinogenesis phase (10 to $40 \mathrm{wps}$ ), the percentage of Tregs to total $\mathrm{CD} 4^{+}$cells was significantly increased in the non-treated controls (Fig. 4g). In contrast, metformin significantly decreased the Treg cell percentages at 20 and 40 wps (Fig. 4g). Similar changes were also observed in 
Th17 cells (Fig. 4h). However, the calculated populations of infiltrated Tregs and Th17 cells in the esophagus at $40 \mathrm{wps}$ were not altered by metformin treatment (Fig. 4g, h). These findings suggest that metformin did not markedly affect the regulation of Treg and Th17 cell numbers. However, TGF$\beta^{+}$and IL $-10^{+}$Tregs were significantly decreased at $40 \mathrm{wps}$ (Fig. 4k).

We next investigated changes in NK and NKT cell populations. NK cells were significantly increased at 10 and 40 wps (inflammatory and carcinogenesis phases, respectively) upon metformin treatment, and the infiltration of NKT cells was significantly increased at all stages in the
Fig. 4 Flow cytometric analyses of characteristics and the number of infiltrating immune cells in the lesions of the esophagus at 10, 20, and 40 weeks $(10 \mathrm{~W}, 20 \mathrm{~W}$, and $40 \mathrm{~W}$, respectively) after the surgery in metformin-treated groups [Met $(+)$, dark shading] and the nontreated controls (Met (-), gray shading). a, b CD3 ${ }^{+} \mathrm{T}$ cells. c-f CD4 ${ }^{+}$ and $\mathrm{CD} 8^{+} \mathrm{T}$ cells. $\mathbf{g}$ Treg cells. $\mathbf{h}$ Th17 cells. $\mathbf{i}, \mathbf{j}$ TNF- $\alpha$ and IFN- $\gamma$ expressions in $\mathrm{CD}^{+} \mathrm{T}$ cells, and $\mathrm{CD} 8^{+} \mathrm{T}$ cells. MFI, mean fluorescence intensity. $\mathbf{l}$ NK and NKT cells. $\mathbf{k}$ TGF- $\beta^{+}$IL- $10^{+}$Treg cells in Treg cells. Fluorescence minus one (FMO) controls were used to set gates. Data are presented as mean $\pm \mathrm{SEM} ; n=6$ per group; *, $p<0.05 ; * *, p<0.01 ; * * *, p<0.001$
Fig. 3 a Macroscopic and microscopic findings of the distal esophagus in non-treated and metformin-treated group at 10,20 , and 40 weeks after the esophagojejunostomy. Wall thickening and dilatation changes of the esophagus as well as the lesion sizes of Barrett's metaplasia and esophageal cancer were attenuated in the metformin-treated group [Met (+)] compared with those in the non-treated control [Met (-)]. Microscopic findings (H\&E stain; magnification, $\times 10)$. b The incidence rate of Barrett's metaplasia and esophageal cancer in rats with or without metformin treatment $[\operatorname{Met}(+)$ or Met (-), respectively] at $10(10 \mathrm{~W}, \mathrm{n}=10), 20(20 \mathrm{~W}$, $n=10), 30(30 \mathrm{~W}, n=10)$, and 40 weeks $(40 \mathrm{~W}, n=30)$ after the esophagojejunostomy. *, $p<0.05$. c The proportion of histological types of esophageal cancer in rats with or without the metformin treatment [Met (+) or Met (-), respectively]. ESCC, esophageal squamous cell carcinoma; EAC, esophageal adenocarcinoma
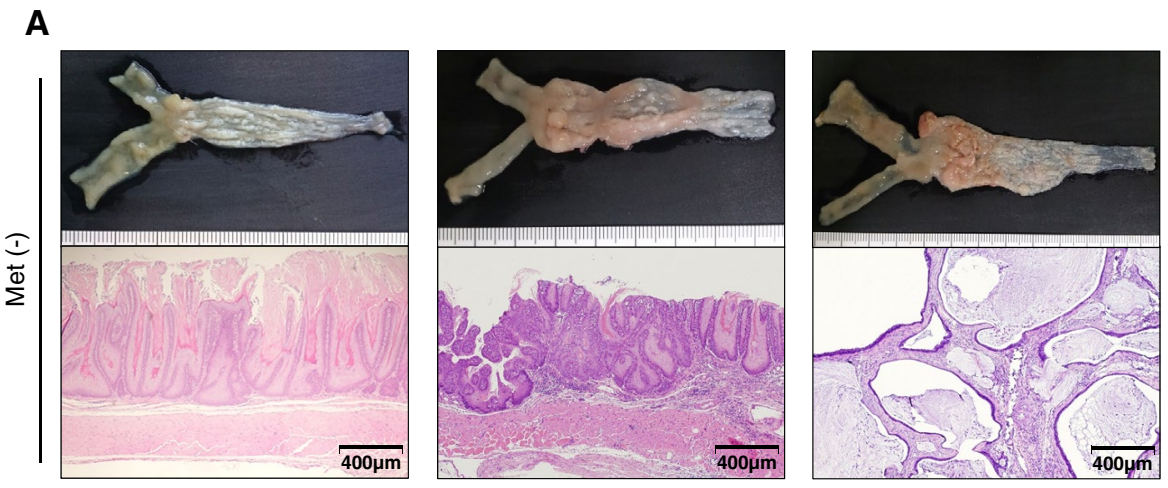

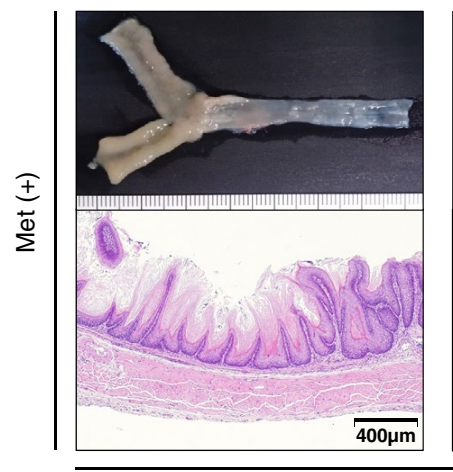

Proliferative hyperplasia

B

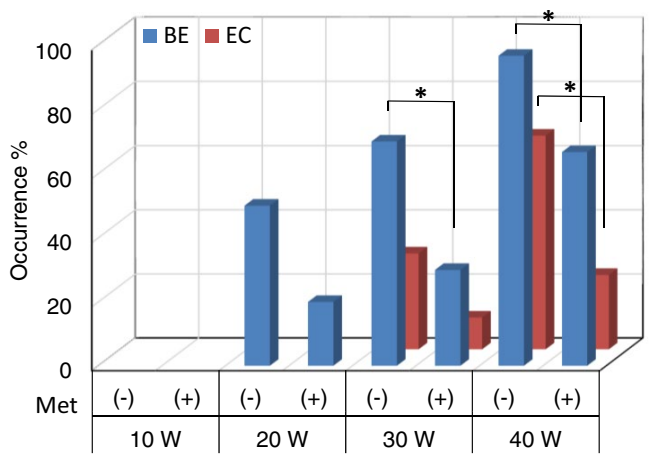

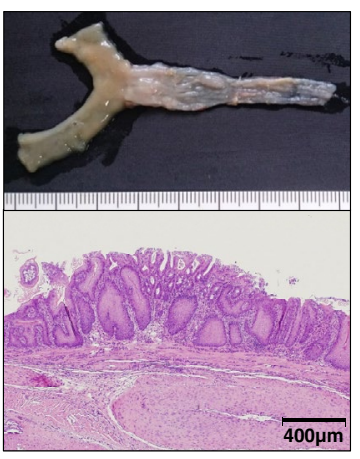

20 weeks

Barrett's metaplasia

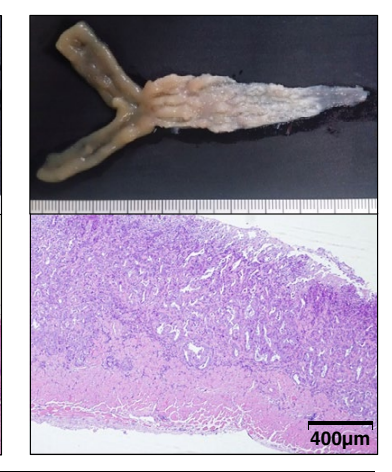

40 weeks

Esophageal cancer
C

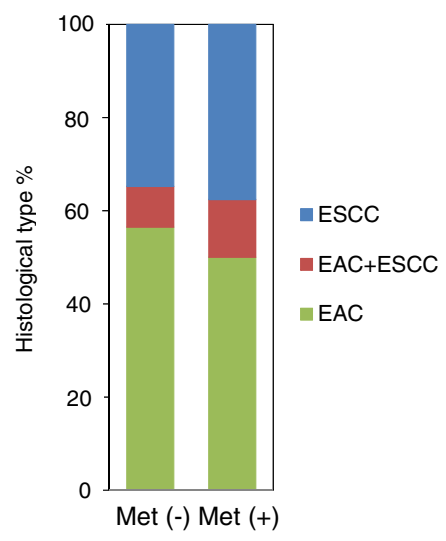



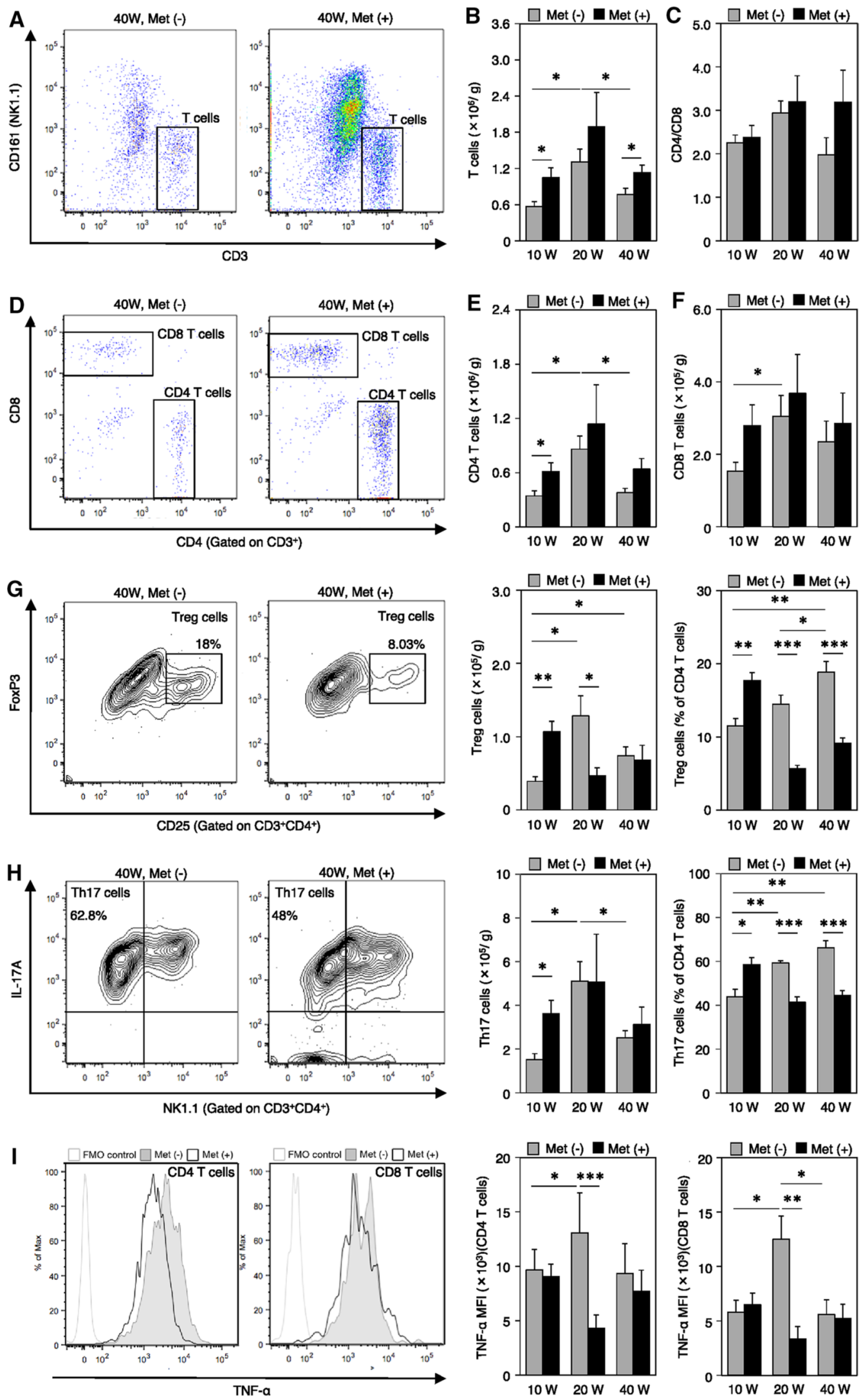

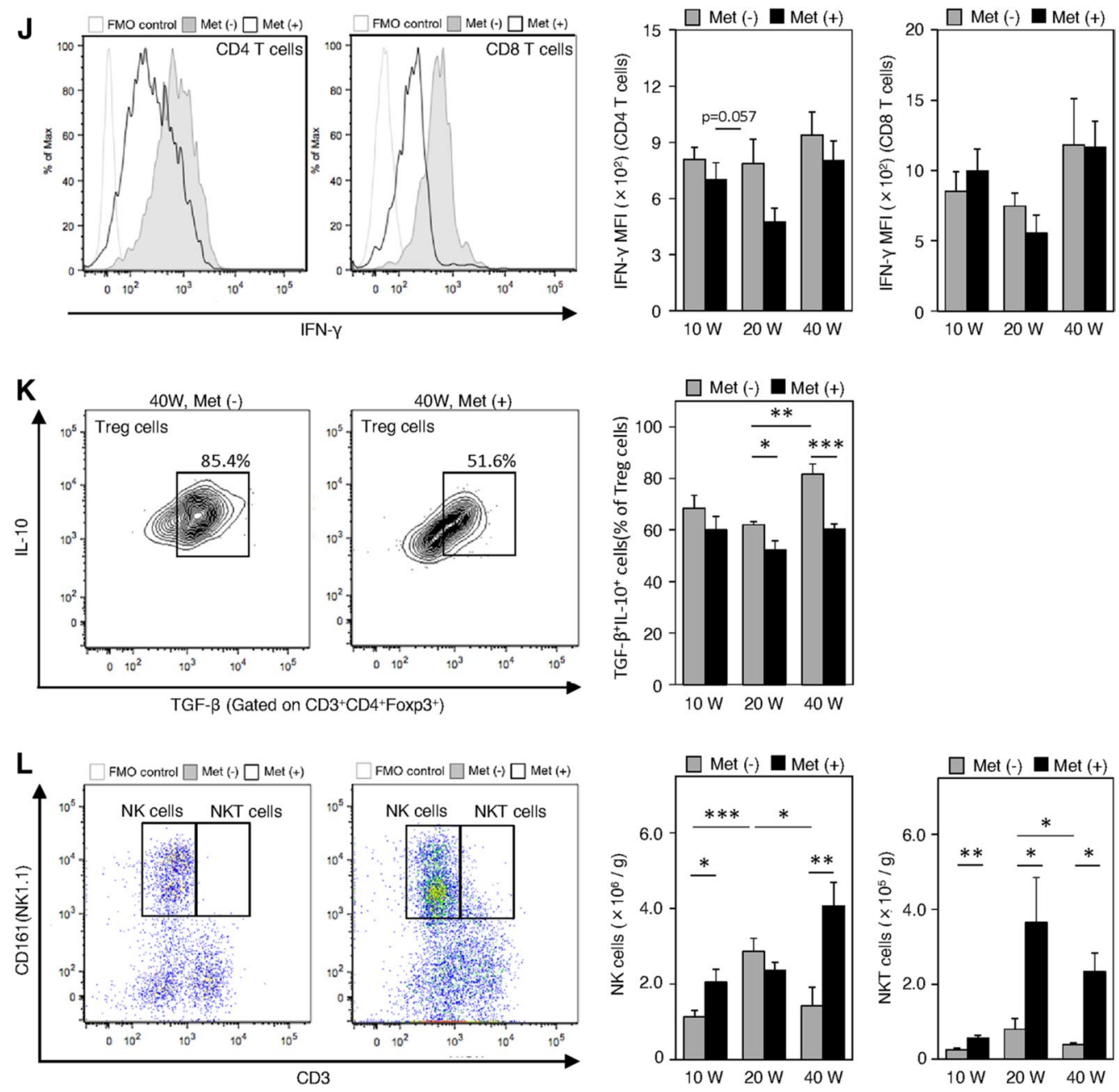

Fig. 4 (continued)

metformin-treated group compared with that in the nontreated group (Fig. 4l).

We next examined changes in the macrophage populations and their characteristics (Fig. 5). The infiltration of $\mathrm{CD} 11 \mathrm{~b}^{+} \mathrm{CD} 68^{+} \mathrm{CD} 86^{+} \mathrm{M} 1 \mathrm{Ms}$ in the esophagus significantly increased at $20 \mathrm{wps}$ relative to that at $10 \mathrm{wps}$, and then significantly decreased at $40 \mathrm{wps}$ in the non-treated group (Fig. 5a). However, metformin treatment significantly upregulated the number of M1Ms at $10 \mathrm{wps}$ (Fig. 5a). This increase was maintained until 40 wps (Fig. 5a). The percentage of $\mathrm{CD} 11 \mathrm{~b}^{+} \mathrm{CD} 68^{+} \mathrm{CD} 86^{+} \mathrm{M} 1 \mathrm{Ms}$ relative to total macrophages was $\sim 50 \%$ until $20 \mathrm{wps}$, but significantly decreased in the carcinogenesis phase in the non-treated group (Fig. 5a). However, metformin did not decrease the infiltration of M1Ms (Fig. 5a). The calculated number of infiltrated M1Ms significantly increased in the esophagus at 10 and $40 \mathrm{wps}$ in the metformin-treated group compared to those in the non-treated control (Fig. 5a). In contrast, the percentage of $\mathrm{CD} 11 \mathrm{~b}^{+} \mathrm{CD} 68^{+} \mathrm{CD} 163^{+} \mathrm{M} 2 \mathrm{Ms}$ relative to total macrophages was increased at 40 wps (carcinogenesis phase) compared to that at $10 \mathrm{wps}$ in the non-treated group, but was significantly reduced by metformin treatment at all stages, especially at $20 \mathrm{wps}$ (Barrett rising phase) (Fig. 5b). The calculated numbers of M2Ms were increased at $10 \mathrm{wps}$ but significantly decreased at $20 \mathrm{wps}$ in the metformintreated group compared to the values in the untreated control group (Fig. 5b). These data showed that metformin treatment shifted the M1Ms/M2Ms balance in favor of M1Ms during the observation period. We then examined the changes in the expression of TNF- $\alpha$ and IFN- $\gamma$ in M1Ms (Fig. 5c), and of TGF- $\beta$ and IL-10 in M2Ms (Fig. $5 \mathrm{~d}$ ). TNF- $\alpha$ expression was downregulated at $20 \mathrm{wps}$ (Barrett rising phase) in the metformin group (Fig. 5c). This change was also apparent at 40 wps compared to that in the non-treated group at 20 wps (Fig. 5c). However, paradoxically, its expression was upregulated at $40 \mathrm{wps}$ in the metformin-treated group (Fig. 5c). IFN- $\gamma$ levels in M1Ms at 40 wps were slightly but significantly decreased upon metformin treatment 
Fig. 5 Flow cytometric analyses of characteristics and the number of macrophages infiltrated in the lesions of the esophagus at 10,20 , and 40 weeks $(10 \mathrm{~W}$, $20 \mathrm{~W}$, and $40 \mathrm{~W}$, respectively) after the surgery in metformintreated groups [Met $(+)$, dark shading] and the non-treated controls (Met (-), gray shading). a, b The number and the percentage of the M1-polarized macrophages (M1Ms) and M2-polarized macrophages (M2Ms). c Expressions of TNF- $\alpha$ and IFN- $\gamma$ in M1Ms. MFI, mean fluorescence intensity. d Expressions of TGF- $\beta$ and IL-10 in M2Ms. MFI, mean fluorescence intensity. e Levels of phosphorylated Stat3 (pStat3) (pTyr705 and pSer727) in macrophages. MFI, mean fluorescence intensity. Fluorescence minus one (FMO) controls were used to set gates. Data are presented as mean \pm SEM; $n=6$ per group; *, $p<0.05$, **, $p<0.01, * * *, p<0.001$
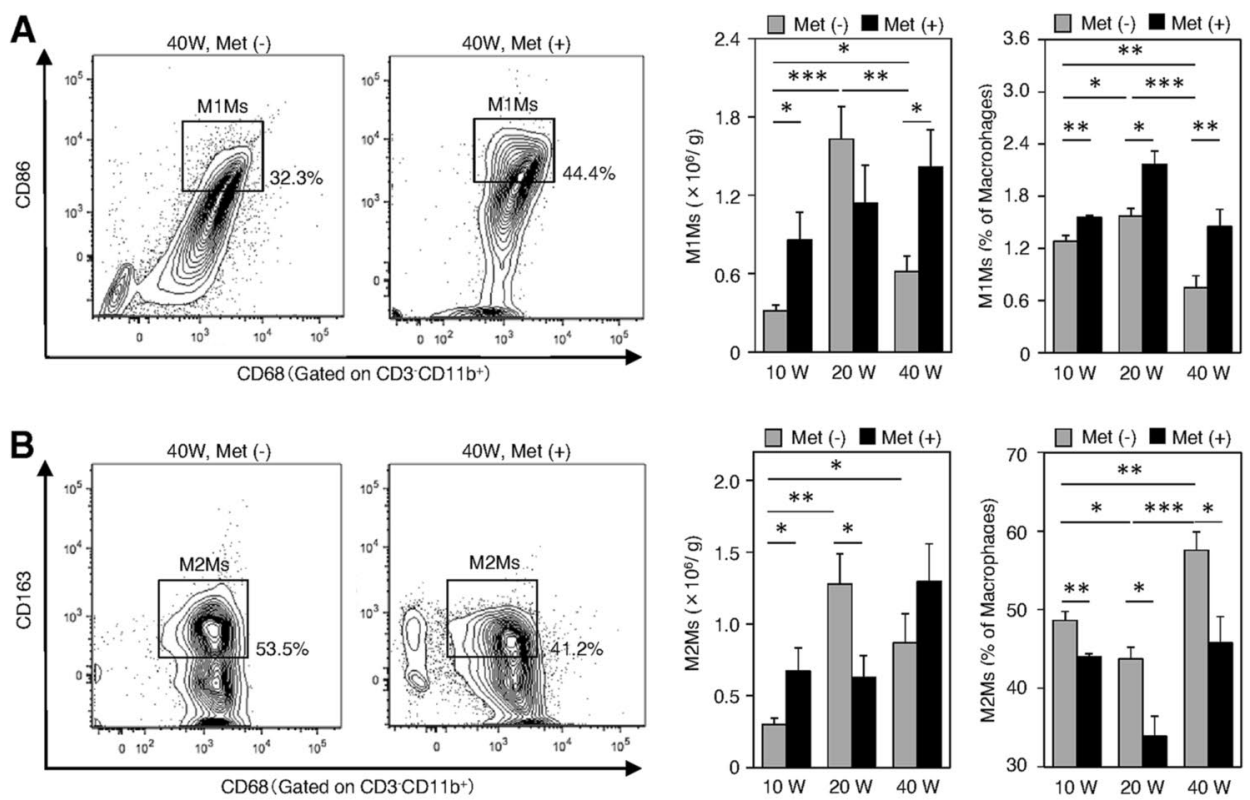

C
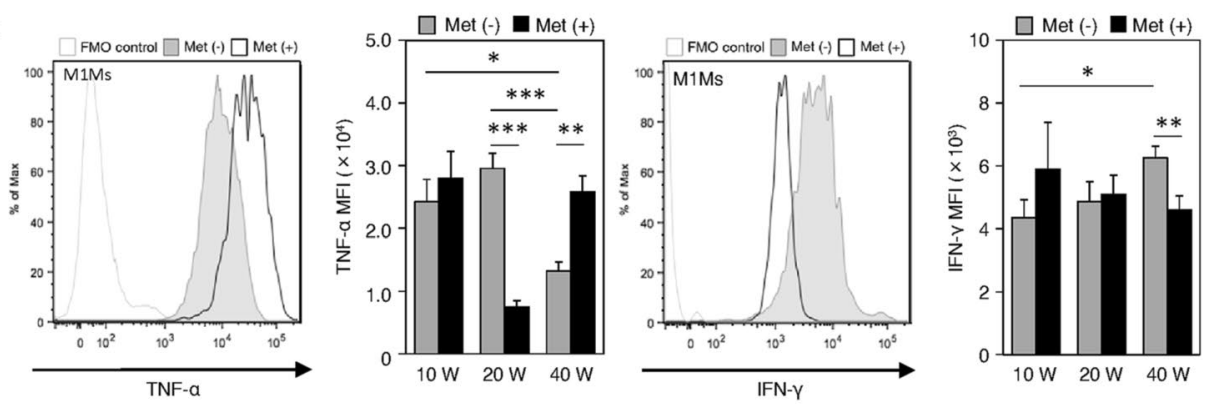

D
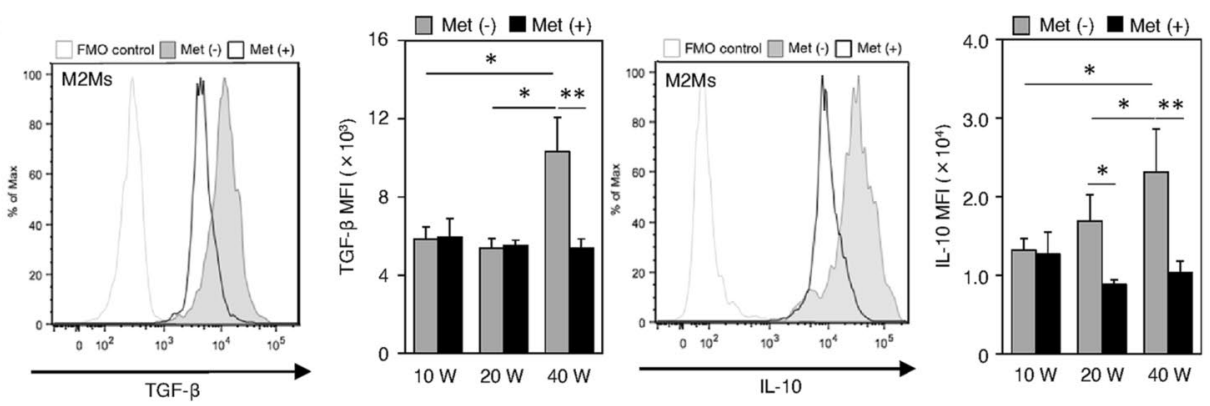

E
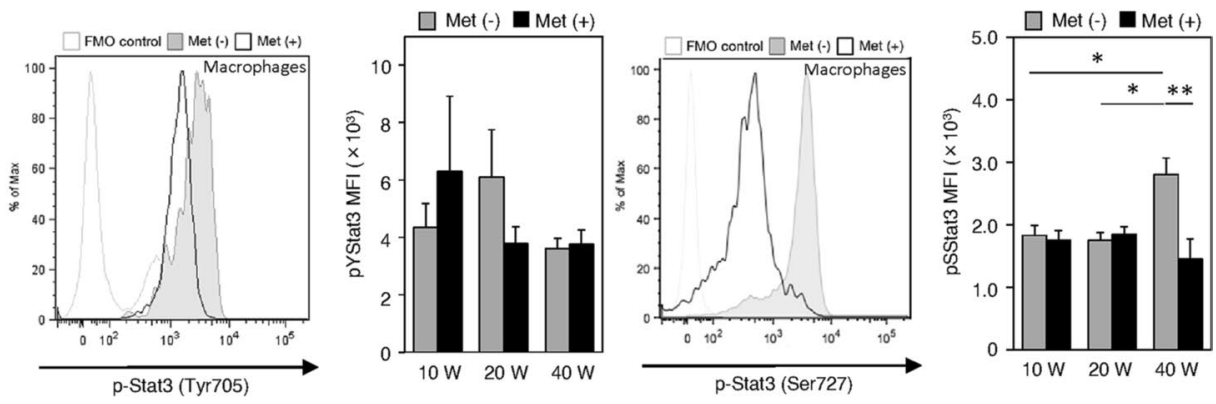

(Fig. 5c). In M2Ms, the expression of TGF- $\beta$ and IL-10 was increased at $40 \mathrm{wps}$ (carcinogenesis phase) (Fig. 5d). These increases were downregulated upon metformin treatment, suggesting that metformin stimulated immunological modulations (Fig. 5d). These findings led us to investigate macrophage p-Stat3 (pTyr705 and pSer727) levels. We could not observe any significant differences in p-Stat3 (pTyr705) levels among the groups (Fig. 5e). The p-Stat3 (pSer727) 
expression patterns in the non-treated control mirrored those of TNF- $\alpha$, consistent with the percentage of M1Ms and similar to the expression patterns of TGF- $\beta$, IL-10, and the percentage of M2Ms (Fig. 5c-e). Metformin treatment inhibited the upregulation of macrophage p-Stat3 (Ser727) levels at 40 wps (Fig. 5e).

\section{Discussion}

Chronic inflammation caused by extrinsic (infection, autoimmune disease, or caustic chemical injury) and intrinsic (genetic alterations) factors is a risk factor for carcinogenesis of the gastrointestinal tract, including EC [22]. In our longstanding rat surgical model, which is triggered by exposure to bile acid and chronic inflammation, EC develops spontaneously due to chronic GERD [13]. This spontaneous carcinogenesis model has morphological and pathological features similar to human clinical EC. The present macroand microscopic examinations revealed the development of $\mathrm{BE}$ in almost all animals at $20 \mathrm{wps}$, followed by EC in $67 \%$ of the experimental animals at 30-40 wps (Fig. 3b). This occurrence rate was almost the same as the rate was reported previously [23].

Cancer cells stimulate and promote the formation of a tumor microenvironment that can suppress antitumor immunity and obtain the nutrients for their growth and progression, in the same manner as in esophageal carcinogenesis [24]. The inhibition of antitumor immunity typically includes an increase in M2Ms and Tregs, accompanied by a decrease in M1Ms, $\mathrm{CD}^{+} \mathrm{T}$, NK, and NKT cells surrounding the tumors $[25,26]$. Previous studies have investigated the difference in the immune composition between normal esophagus, reflux esophagitis, BE, and EC using human biopsies or mouse models [27-29]. In addition to the analyses of the dynamic changes in the immune composition of chronic inflammation on reflux esophagitis and the tumor microenvironment of EC using the rat surgical carcinogenesis model, we examined the immunomodulating effect of metformin, which shows inhibitory activity against STAT3. Our overall findings across all phases of esophageal carcinogenesis and the dynamic changes in the populations and characteristics of immune cells with or without metformin treatment in this model are summarized in Fig. 6.

Under baseline natural conditions, $\mathrm{CD} 4^{+} \mathrm{T}$ cells, $\mathrm{NK}$ cells, and both M1Ms and M2Ms were detectable in the esophagus (Fig. 2). In particular, the immunosuppressive Treg cell population was predominant (Fig. 4). $\mathrm{CD} 8^{+} \mathrm{T}$ and NKT cells were rarely observed (Fig. 2). Tissue resident
Treg cells and macrophages are essential for gut homeostasis because they regulate the mucosal immune response [30, 31]. After the onset of reflux esophagitis, both pro- and anti-inflammatory cells were recruited to the esophagus. The former included $\mathrm{CD} 4^{+} \mathrm{T}$, Th17, $\mathrm{CD} 8^{+} \mathrm{T}, \mathrm{M} 1 \mathrm{Ms}, \mathrm{NK}$, and NKT cells. The latter included Treg and M2M cells. These inflammatory reactions were strongly promoted and peaked at $20 \mathrm{wps}$ in esophagitis (Barrett rising phase). During this phase, the NK cell population was markedly increased and TNF- $\alpha$ levels in $\mathrm{CD} 4^{+}$and $\mathrm{CD} 8^{+} \mathrm{T}$ cells were upregulated (Fig. 41, i). At 40 wps, during the carcinogenesis phase, the proportions of tumor-suppressive cells that included M1M, NK, and NKT cells were reduced, while those of tumorpromoting cells that included M2Ms and Treg cells were less affected (Figs. 4, 5). However, from a functional perspective, a significant increase in the ratio of TGF- $\beta$ and IL-10 positive cells in Treg cells, the marked elevation of the expression of TGF- $\beta$ and IL-10 in M2Ms, and the notably higher phosphorylation of STAT3 (Ser727) in macrophages was noted (Figs. 4, 5). A previous report described that IL10-mediated activation of STAT3 promoted M2-polarization in macrophages [32]. These findings demonstrated that the tumor microenvironment shifts from a more immune-suppressive or immune evasion in the period from the Barrett rising phase to the carcinogenesis phase [25].

In terms of the effects of metformin, the populations of both pro- and anti-inflammatory cells were increased in the reflux esophagitis phase compared to those in the untreated control (Figs. 4, 5). At $20 \mathrm{wps}$, the NKT cell population was expanded and, in contrast, Treg cell expansion was largely inhibited and the levels of immunosuppressive cytokines were decreased (Fig. 4). The findings are compatible with previous reports showing that metformin inhibited Treg cell differentiation and function by reducing FoxP3 expression $[33,34]$. Furthermore, metformin downregulated the expression of TNF- $\alpha$ in $\mathrm{CD}^{+} \mathrm{T}$ and M1M cells (Figs. 4, 5). The immune cell contents and patterns in untreated controls at 20 wps closely resembled those in the metformin-treated esophagus samples at $40 \mathrm{wps}$, suggesting that metformin treatment delayed the development of esophageal carcinogenesis as a chemopreventive drug.

In summary, we provide the first evidence of dynamic changes in the infiltration of immune cells during the carcinogenic transition from GERD to BM and finally to EC in the esophagus. The results show that immunomodulating agents, such as metformin, could potentially serve as preventive and therapeutic strategies against esophageal carcinogenesis. 
Fig. 6 Illustrated here are dynamic changes of immune cell populations and characteristics during the carcinogenic transition from gastroesophageal reflux disease (GERD) to Barrett's esophagus (BE) and finally to esophageal cancer (EC) in the esophagus of this rat model. Inflammatory reaction is initiated around 10 weeks after the surgery and proliferative hyperplasia (PHP) of the esophagus epithelial cells is observed. Metformin could impact the modulation of pro-inflammatory reactions in esophageal carcinogenesis and host antitumor immunity by improving the immunosuppressive tumor microenvironment and immune evasion
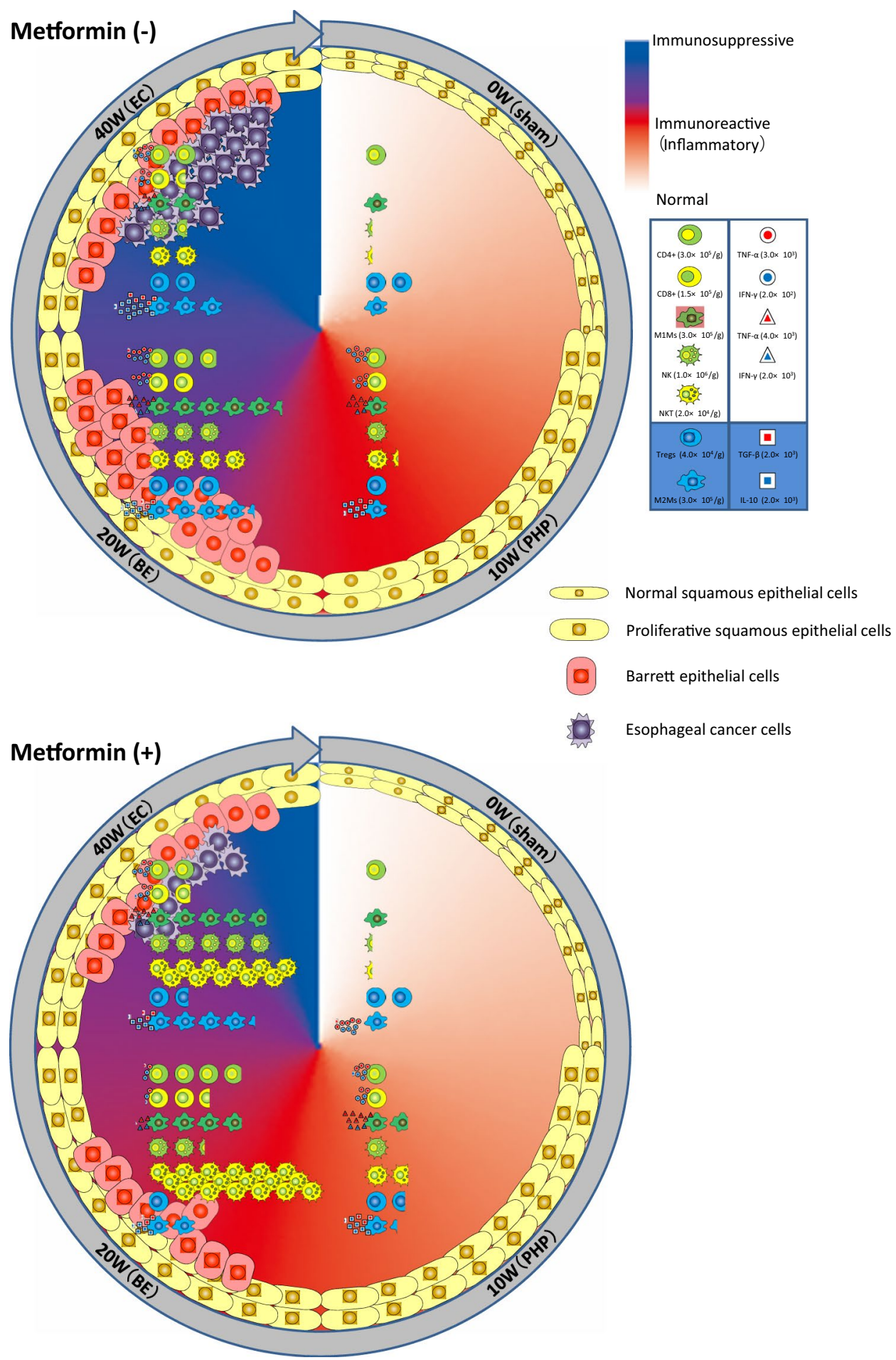

Acknowledgements We thank Y. Niimura and A. Katayama for technical assistance. The document was edited by Editage, a division of Cactus Communications

Authors' contributions RT, TM, TO, and YY conceived the study and study design; RT, ST, AH, SM, SY, NI, and YY performed the experiments and data analysis; RT and YY wrote the paper; HT, IN, HT,
SF, SY, NI, and TO discussed the paper. All of the authors approved this paper.

Funding We acknowledge the financial support from Kanazawa University SAKIGAKE project 2018, Takeda Science Foundation, and Grants-in-Aid for Scientific Research (18K07551, 20K07323, and 18K06889) and the Program for Fostering Globally Talented Researchers from the Japan Society for Promotion of Sciences. 


\section{Declarations}

Conflict of interest The authors have no conflicts of interest to report.

Open Access This article is licensed under a Creative Commons Attribution 4.0 International License, which permits use, sharing, adaptation, distribution and reproduction in any medium or format, as long as you give appropriate credit to the original author(s) and the source, provide a link to the Creative Commons licence, and indicate if changes were made. The images or other third party material in this article are included in the article's Creative Commons licence, unless indicated otherwise in a credit line to the material. If material is not included in the article's Creative Commons licence and your intended use is not permitted by statutory regulation or exceeds the permitted use, you will need to obtain permission directly from the copyright holder. To view a copy of this licence, visit http://creativecommons.org/licenses/by/4.0/.

\section{References}

1. Rustgi AK, El-Serag HB (2014) Esophageal carcinoma. N Engl J Med 371:2499-2509. https://doi.org/10.1056/nejmra1314530

2. Solaymani-Dodaran M, Logan RF, West J, Card T, Coupland C (2004) Risk of oesophageal cancer in Barrett's oesophagus and gastro-oesophageal reflux. Gut 53:1070-1074. https://doi.org/10. 1136/gut.2003.028076

3. Murata M, Thanan R, Ma N, Kawanishi S (2012) Role of nitrative and oxidative DNA damage in inflammation-related carcinogenesis. J Biomed Biotechnol 2012:623019. https://doi.org/10.1155/ 2012/623019

4. Chung SM, Kao J, Hyjek E, Chen Y (2007) p53 in esophageal adenocarcinoma: a critical reassessment of mutation frequency and identification of $72 \mathrm{Arg}$ as the dominant allele. Int $\mathrm{J}$ Oncol 31:1351-1355. https://doi.org/10.3892/ijo.31.6.1351

5. Hardie LJ, Darnton SJ, Wallis YL, Chauhan A, Hainaut P, Wild CP, Casson AG (2005) p16 expression in Barrett's esophagus and esophageal adenocarcinoma: association with genetic and epigenetic alterations. Cancer Lett 217:221-230. https://doi.org/10. 1016/j.canlet.2004.06.025

6. Boonstra JJ, van Marion R, Douben HJ, Lanchbury JS, Timms KM, Abkevich V, Tilanus HW, de Klein A, Dinjens WN (2012) Mapping of homozygous deletions in verified esophageal adenocarcinoma cell lines and xenografts. Genes Chromosomes Cancer 51:272-282. https://doi.org/10.1002/gcc.20952

7. Phillips WA, Russell SE, Ciavarella ML, Choong DY, Montgomery KG, Smith K, Pearson RB, Thomas RJ, Campbell IG (2006) Mutation analysis of PIK3CA and PIK3CB in esophageal cancer and Barrett's esophagus. Int J Cancer 118:2644-2646. https://doi. org/10.1002/ijc.21706

8. Pühringer-Oppermann FA, Stein HJ, Sarbia M (2007) Lack of EGFR gene mutations in exons 19 and 21 in esophageal (Barrett's) adenocarcinomas. Dis Esophagus 20:9-11. https://doi.org/ 10.1111/j.1442-2050.2007.00630.x

9. Choi YW, Heath EI, Heitmiller R, Forastiere AA, Wu TT (2000) Mutations in beta-catenin and APC genes are uncommon in esophageal and esophagogastric junction adenocarcinomas. Mod Pathol 13:1055-1059. https://doi.org/10.1038/modpathol.38801 94

10. Catlett-Falcone R, Landowski TH, Oshiro MM, Turkson J, Levitzki A, Savino R, Ciliberto G, Moscinski L, Fernández-Luna JL, Nuñez G, Dalton WS, Jove R (1999) Constitutive activation of Stat3 signaling confers resistance to apoptosis in human U266 myeloma cells. Immunity 10:105-115. https://doi.org/10.1016/ s1074-7613(00)80011-4
11. Hossain DM, Panda AK, Manna A, Mohanty S, Bhattacharjee P, Bhattacharyya S, Saha T, Chakraborty S, Kar RK, Das T, Chatterjee S, Sa G (2013) FoxP3 acts as a cotranscription factor with STAT3 in tumor-induced regulatory T cells. Immunity 39:10571069. https://doi.org/10.1016/j.immuni.2013.11.005

12. Yuan F, Fu X, Shi H, Chen G, Dong P, Zhang W (2014) Induction of murine macrophage $\mathrm{M} 2$ polarization by cigarette smoke extract via the JAK2/STAT3 pathway. PLoS ONE 9:e107063. https://doi. org/10.1371/journal.pone.0107063

13. Miyashita T, Ohta T, Fujimura T, Ninomiya I, Fushida S, Hattori T, Miwa K (2006) Duodenal juice stimulates oesophageal stem cells to induce Barrett's oesophagus and oesophageal adenocarcinoma in rats. Oncol Rep 15:1469-1475. https://doi.org/10.3892/ or.15.6.1469

14. Libby G, Donnelly LA, Donnan PT, Alessi DR, Morris AD, Evans JM (2009) New users of metformin are at low risk of incident cancer: a cohort study among people with type 2 diabetes. Care 32:1620-1625. https://doi.org/10.2337/dc08-2175

15. A registry and results database of publicly and privately supported clinical studies of human participants conducted around the world, Available online: https://clinicaltrials.gov/. Accessed 8 September 2019

16. Florio T (2015) Antitumoral effects of metformin on cancer stem cells. Ann d'Endocrinol. https://doi.org/10.1016/j.ando.2015.07. 021

17. Hirsch HA, Iliopoulos D, Tsichlis PN, Struhl K (2009) Metformin selectively targets cancer stem cells, and acts together with chemotherapy to block tumor growth and prolong remission. Cancer Res 69:7507-7511. https://doi.org/10.1158/0008-5472.can-09-2994

18. Shank JJ, Yang K, Ghannam J, Cabrera L, Johnston CJ, Reynolds RK, Buckanovich RJ (2012) Metformin targets ovarian cancer stem cells in vitro and in vivo. Gynecol Oncol 127:390-397. https://doi.org/10.1016/j.ygyno.2012.07.115

19. Feng Y, Ke C, Tang Q, Dong H, Zheng X, Lin W, Ke J, Huang J, Yeung SC, Zhang H (2014) Metformin promotes autophagy and apoptosis in esophageal squamous cell carcinoma by downregulating Stat3 signaling. Cell Death Dis 5:e1088. https://doi.org/10. 1038/cddis.2014.59

20. Yue W, Zheng X, Lin Y, Yang CS, Xu Q, Carpizo D, Huang H, DiPaola RS, Tan XL (2015) Metformin combined with aspirin significantly inhibit pancreatic cancer cell growth in vitro and in vivo by suppressing anti-apoptotic proteins Mcl-1 and Bcl-2. Oncotarget. 6:21208-21224. https://doi.org/10.18632/oncotarget. 4126

21. Deng XS, Wang S, Deng A, Liu B, Edgerton SM, Lind SE, Wahdan-Alaswad R, Thor AD (2012) Metformin targets Stat3 to inhibit cell growth and induce apoptosis in triple-negative breast cancers. Cell Cycle 11:367-376. https://doi.org/10.4161/cc.11.2. 18813

22. Shacter E, Weitzman SA (2002) Chronic inflammation and cancer. Oncology (Williston Park) 16:217-226

23. Miyashita T, Tajima H, Shah FA, Oshima M, Makino I, Nakagawara H, Kitagawa H, Fujimura T, Harmon JW, Ohta T (2014) Impact of inflammation-metaplasia-adenocarcinoma sequence and inflammatory microenvironment in esophageal carcinogenesis using surgical rat models. Ann Surg Oncol 21:2012-2019. https://doi.org/10.1245/s10434-014-3537-5

24. Hanahan D, Weinberg RA (2011) Hallmarks of cancer: the next generation. Cell 144:646-674. https://doi.org/10.1016/j.cell.2011. 02.013

25. Burkholder B, Huang RY, Burgess R, Luo S, Jones VS, Zhang W, Lv ZQ, Gao CY, Wang BL, Zhang YM, Huang RP (2014) Tumorinduced perturbations of cytokines and immune cell networks. Biochim Biophys Acta 1845:182-201. https://doi.org/10.1016/j. bbcan.2014.01.004 
26. Mamessier E, Sylvain A, Thibult ML, Houvenaeghel G, Jacquemier J, Castellano R, Gonçalves A, André P, Romagné F, Thibault G, Viens P, Birnbaum D, Bertucci F, Moretta A, Olive D (2011) Human breast cancer cells enhance self tolerance by promoting evasion from NK cell antitumor immunity. J Clin Invest 121:3609-3622. https://doi.org/10.1172/jci45816

27. Lind A, Koenderman L, Kusters JG, Siersema PD (2014) Squamous tissue lymphocytes in the esophagus of controls and patients with reflux esophagitis and Barrett's esophagus are characterized by a non-inflammatory phenotype. PLoS ONE 9:e106261. https:// doi.org/10.1371/journal.pone.0106261

28. Sen M, Hahn F, Black TA, DeMarshall M, Porter W, Snowden E, Yee SS, Tong F, Ferguson M, Fleshman EN, Nakagawa H, Falk GW, Ginsberg GG, Kochman ML, Blaesius R, Rustgi AK, Carpenter EL (2019) Flow based single cell analysis of the immune landscape distinguishes Barrett's esophagus from adjacent normal tissue. Oncotarget. 10:3592-3604. https://doi.org/10.18632/oncot arget.26911

29. Kavanagh ME, Conroy MJ, Clarke NE, Gilmartin NT, O'Sullivan KE, Feighery R, MacCarthy F, O'Toole D, Ravi N, Reynolds JV, O'Sullivan J, Lysaght J (2016) Impact of the inflammatory microenvironment on $\mathrm{T}$-cell phenotype in the progression from reflux oesophagitis to Barrett oesophagus and oesophageal adenocarcinoma. Cancer Lett 370:117-124. https://doi.org/10.1016/j. canlet.2015.10.019
30. Luu M, Steinhoff U, Visekruna A (2017) Functional heterogeneity of gut-resident regulatory T cells. Clin Transl Immunol 6:e156. https://doi.org/10.1038/cti.2017.39

31. Muller PA, Matheis F, Mucida D (2020) Gut macrophages: key players in intestinal immunity and tissue physiology. Curr Opin Immunol 62:54-61. https://doi.org/10.1016/j.coi.2019.11.011

32. Nakamura R, Sene A, Santeford A, Gdoura A, Kubota S, Zapata N, Apte RS (2015) IL10-driven STAT3 signalling in senescent macrophages promotes pathological eye angiogenesis. Nat Commun 6:7847. https://doi.org/10.1038/ncomms8847

33. Ono M, Yaguchi H, Ohkura N, Kitabayashi I, Nagamura Y, Nomura T, Miyachi Y, Tsukada T, Sakaguchi S (2007) Foxp3 controls regulatory T-cell function by interacting with AML1/ Runx1. Nature 446:685-689. https://doi.org/10.1038/nature05673

34. Kunisada Y, Eikawa S, Tomonobu N, Domae S, Uehara T, Hori S, Furusawa Y, Hase K, Sasaki A, Udono H (2017) Attenuation of CD4+CD25+ Regulatory T Cells in the tumor microenvironment by metformin, a Type 2 Diabetes Drug. EBioMedicine 25:154164. https://doi.org/10.1016/j.ebiom.2017.10.009

Publisher's Note Springer Nature remains neutral with regard to jurisdictional claims in published maps and institutional affiliations. 